\title{
Investigation of the behaviour of functionally graded materials under impact loads
}

\author{
Maxim Orlov ${ }^{1}$, Yuri Orlov ${ }^{1}$, Viktor Glazyrin ${ }^{1}$ and Yulia Orlova $^{2}$ \\ ${ }^{1}$ Tomsk State University, 634050, Tomsk, Russia \\ ${ }^{2}$ Tomsk Polytechnic University, 634050, Tomsk, Russia
}

\begin{abstract}
The impact response of unidirectional FGM is studied in detail. The behaviour of the material is described by the classical complex model of continuum mechanics. The calculations are performed in 2D axisymmetric approach using a modified Lagrangian method. The mathematical model and numerical method was developed at the Research Institute of Applied Mathematics and Mechanics of Tomsk State University. In the beginning, test calculations were carried out. The evolution of the destruction of homogeneous and FGM-samples, including the time of formation of the first foci of destruction, spall was studied. The time dependences of the velocity of the free surface, hydrostatic pressure, and the damage parameter were constructed. The presence of a graded substrate led to an increase in impact resistance at velocities up to $275 \mathrm{~m} / \mathrm{s}$.
\end{abstract}

\section{Introduction}

At present time, functional graded materials (hereinafter FGM) are used in power engineering, engine building, medicine, aviation industry, etc. They can transform the incident pulse, including pulse loosen and redistribute in the desired direction of volume, delay in time. This is unique abilities of FGM, which are absent in constructional materials. Nowadays steel, aluminum, titanium and ceramics are used for one production. Computer modelling FGM lags behind the one practical development. Its impact response one have been poorly studied under shock loading conditions [1-2].

Modern technologies of powder metallurgy allow creating materials with a smooth change of properties.

To design new protective structures, materials with a gradient distribution of properties over the cross-section are needed. Therefore, it makes sense to investigate samples with a gradient distribution of strength properties. Samples with a gradient distribution of shear modulus, porosity and strength may well be objects of research [3].

In the current research impact of a steel plate on FGM barriers at low initial velocities were investigated. Only thin barrier of unidirectional FGM are considered as research objects. All samples had gradient substrate disposed at the back side.

\section{Investigation FGM under dynamic loading}

The behaviour of FGM under shock-wave loads is studied in [3]. The previous research functional graded steel samples were investigated. The impact response barrier to the variation along the entire thickness of the sample of the strength characteristics responsible for the formation "shear" and "spallation" fractures under shock wave loading has been studied. The interval of variation in strength characteristics corresponded to real steel alloys. It was considered unidirectional FGM, bidirectional FGM, and homogeneous steel barriers at shockwave loading. The process of destruction of barriers under loading by shockwave and under impacted by cylinder and projectile is studied in detail. The results obtained allowed us to formulate new research tasks that were solved in the current research.

\subsection{Investigation of unidirectional and bi- directional FGM under shock-wave loading}

This section focuses on the results of FGM numerical simulation under shock wave loading. As describes in previous section, both unidirectional and bidirectional FGM based on Steel 3 were considered. The subject of research was effect of a linear change two strength characteristics throughout the entire thickness of the plate. The first characteristic $\sigma_{k}$ is responsible for the formation of "spall" fractures. The second characteristic is called specific work of shear plastic deformations $A_{p}^{*}$.

It is responsible for the formation "shift" fractures. The strength characteristics varied from $2.1 \mathrm{GPa}$ to 3.5 $\mathrm{GPa}$ and from $35 \mathrm{~kJ} / \mathrm{kg}$ to $55 \mathrm{~kJ} / \mathrm{kg}$, respectively. The front side of the sample corresponds to low-strength steel, and the back side of the sample corresponds to high-strength steel. Strength characteristics for a homogeneous sample were as follows: $\sigma_{k}=2.8 \mathrm{GPa}$ $A_{p}^{*}=45 \mathrm{~kJ} / \mathrm{kg}$.

In total, nine samples were considered, including a homogeneous sample. For convenience, the following 
indices are introduced: '-', '个', ' $\downarrow$ ', which means, respectively, the constancy, increase and decrease of the specified characteristic over the thickness of the barrier. For example, if the barrier is denoted $\left[\sigma_{k}^{-}, A_{k}^{-}\right]$, then the entire thickness of the barrier does not change, i.e. the barrier is homogeneous. In addition, a homogeneous barrier should be considered as the base case. If one is designated as $\left[\sigma_{k}^{\uparrow}, A_{k}^{\downarrow}\right]$, then $\sigma_{k}$ is increases, and $A_{p}^{*}$ is decreases from the loading surface to the rear surface. Indeed, it was one of two bidirectional FGM-samples. The remaining seven barriers are similarly indicated.

Fig. 1 shows the results of numerical modelling of the loading of plane shock wave of homogeneous sample. At $20 \mu \mathrm{s}$ the spall plate is formed, but it is not separated from the sample yet. The rear surface takes the S-shape.

The numerical results were as follows. Shear strength $\left(A_{p}^{*}\right)$ did not affect the destruction of gradient samples when loaded by a shock wave. The main contribution to the process of destruction made spall strength $\left(\sigma_{k}\right)$. During the penetration process of FGM-barriers by a compact and elongated impactor, the main contribution was made by shearing strength $\left(A_{p}^{*}\right)$. For example, the greatest decrease in the speed of the impactor was noted with an increase in shear strength from the face to the rear.

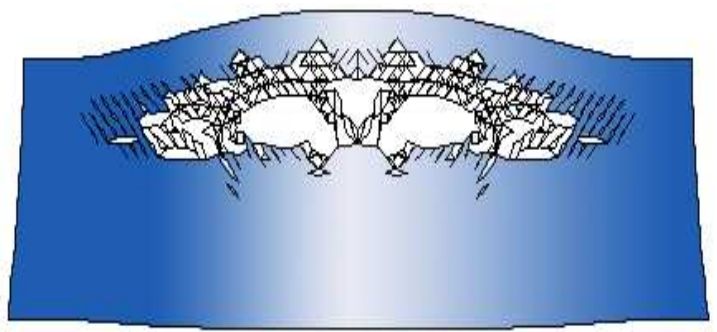

Fig. 1. Steel sample $\left[\sigma_{k}^{-}, A_{k}^{-}\right]$after shockwave load at $t=20$ $\mu \mathrm{s}$. Figure reprinted from [3]. Calculations were obtained using a computer program developed by Yuri Orlov.

Based on the foregoing, it makes sense to replace the gradient sample in the steel samples with a gradient substrate. The thickness of the substrate can vary from $3 / 4$ to $4 / 6$ of the thickness of the entire sample.

\section{Computational method and results}

The first part of the section deals with computational method. In the Scientific Research Institute of Applied Mathematics and Mechanics at Tomsk State University, research on the behaviour of structurally heterogeneous materials under shock and explosive loads are systematically conducted.

Based on the classical complex model of continuum mechanics, a physics and mathematical model of deformation and fracture of structurally inhomogeneous materials under shock and explosive loads have been developed. The model corresponds to modern concepts of destruction of materials under dynamic loads. A numerical Lagrangian method for solving dynamic multi-contact tasks of mechanics of a deformed solid is modified. An experimental base of such studies is being developed, including an experimental set-up with highprecision devices for recording fast processes and an expanding relational database on the behaviour of materials under impact loads.

\subsection{Computational method}

The system of equations for $2 \mathrm{D}$ axisymmetric approach is solved by the modified method of Johnson G.R [4, 5]. A well-known fact this method based on Lagrangian approach to the description of the motion of continuous media. A well-known fact is that any Lagrangian method has problems with penetration and perforating tasks, including deep penetrating modern safety structure. To overcome this lack, the algorithm destruction of triangulation elements, algorithm splitting nodes, the algorithm for constructing the free surface were introduced. According to the terminology [6], the numerical method contains a new way for isolating the surfaces of discontinuity of materials. A similar approach to modeling the perforation tasks developed in the work [7]. However, in the present approach, several ways of splitting nodes are possible. This allows us to use various failure criteria of solids.

The latest innovation should be considered smoothing algorithm for the contact surface. This algorithm can more accurately describe the contact and the free surface between the detonation products and other materials. The presence of this algorithm allows obtain a smooth contact surface and thereby simulate the current task of shock and explosive loading materials [8].

\subsection{Mathematical model and test results}

The governing equations are based on the fundamental laws of conservation of mass, momentum and energy. A complex model of continuum mechanics used to describe the material behaviour under dynamic load. Material modelled a porous, compressible medium, taking into account the strength properties, shock-wave phenomena, as well as formation fracture material [5-6].

To describe the shear strength of a body, the Prandtl - Reuss equations and the von Mises yield conditions were used [8]. The state equation Mi-Grüneisen was used [9]. The mathematical model allows us to use various equations of state, including the wide-range equation of state, etc. In the process of material destruction under dynamic loading, new free surfaces, including fragmentary destruction are allowed.

In the calculation of fracture material, the concept of joint formation fracture like spall and fractures like shear is realized. This makes it possible to simulate the process of the most close to real. The failure criterions were strength characteristics referred in sect.2.1 [10].

\subsection{Test computation}


In the beginning, impact of the steel cylinder on the aluminum plate was modelled. This task should be considered as a test. The initial data of task were as follows:

-Initial velocity of the impactor is $781 \mathrm{~m} / \mathrm{s}$,

- Straight circular cylinder diameter is $8.4 \mathrm{~mm}$,

-Plate thickness is $16.2 \mathrm{~mm}$,

-Impactor material is Steel (Steel 3),

-Target material is Aluminum (D16),

-The total number of elements is 10000 elements.

Subject of comparison was the velocity of the free surface and the diameter of the hole in the aluminum plate after impact. Post penetration analysis consisted of comparing deformation patterns of material destruction, including first foci of destruction, evolution macro cracks, etc. The experimental results are taken from [11].

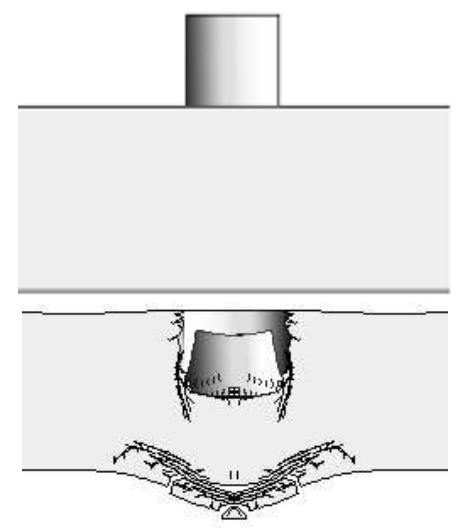

Fig. 2. Steel impactor and aluminum plate before $(t=0 \mu \mathrm{s})$ and after impact $(t=30 \mu \mathrm{s})$. Fragment initial and current configurations. Experimental data by A.Bragov and V. Zaharov.

Free surface velocity profile was constructed also. Fig. 3 shows the velocity profile obtained in the ballistic impact and in the numerical calculations.

In addition, some impact experiments were simulated from work [11-12]. Good agreement between experiment and numerical simulation is observed.

\section{FGM's impact simulation results and discussion}

In this section we will discuss the results of modelling a plate impact on a homogeneous barrier and FGM barrier. The impactor material and barriers were structural carbon Steel 3 (According to the Russian nomenclature of structural material).

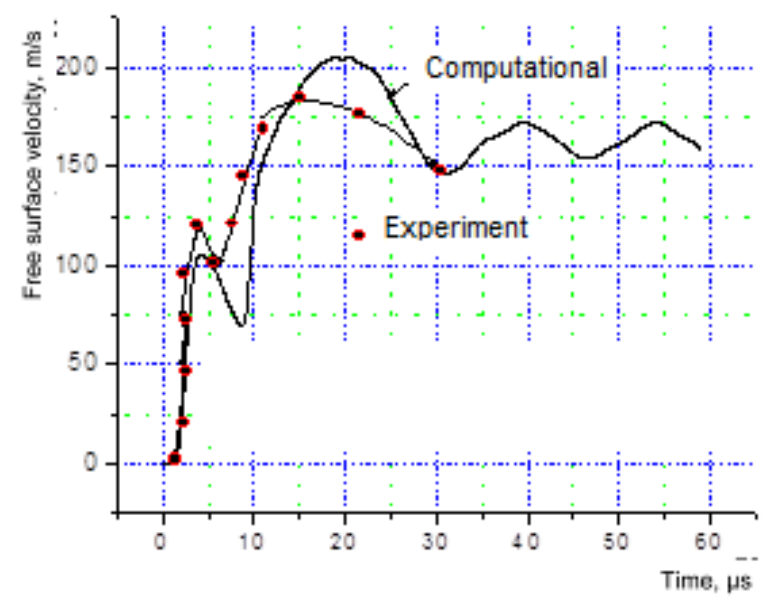

Fig. 3. Free surface velocity profile (experiment Vs computational).

The height of the impactor was $0.4 \mathrm{~cm}$, and the diameter was $4 \mathrm{~cm}$. The calculations were carried out in 2D axisymmetric approach on a PC (Frequency $2 \mathrm{GHz}$ ). The penetrating process was simulated up to $25 \mu \mathrm{s}$, because after the deformation pattern and the area of material destruction did not change. After impact, the steel plate was subjected to plastic deformation. In the current research, the state of the impactor has not been studied in detail.

Fig. 4 shows the initial configuration of the impactor and barrier. On the contact boundary of the impactor and barrier, a sliding condition without friction is specified. In gradations of gray color, a gradient substrate is shown. A total of 6 control points were chosen, the first and last points being in the homogeneous parts of the barrier. The distance between ones was $2.1 \mathrm{~mm}$. At these points, the hydrostatic pressure was calculated only. The direction of the velocity vector coincided with the axis of symmetry of the sample.

As it was said above research objects are homogeneous barrier and steel barrier with a graded substrate. The height of the sample is $1.4 \mathrm{~cm}$, diameter is $4 \mathrm{~cm}$. The thickness of the graded substrate is $8.6 \mathrm{~mm}$, i.e. $4 / 6$ heights of the barrier. The domain was divided by 20000 triangulation elements. The initial impactor velocity varied from 225 to $300 \mathrm{~m} / \mathrm{s}$. Spalling strength $\left(\sigma_{\mathrm{k}}\right)$ in the substrate increased from the front to the rear and deep into $1 / 6$ from the front to the rear and from the rear to the front sample was homogeneous. A sample with a gradient substrate will be denoted by $\left[\frac{4}{6} \sigma_{k}^{\uparrow}, A_{p}\right]$.The minimum spall strength was $2.1 \mathrm{GPa}$, and the maximum spall strength was $3.5 \mathrm{GPa}$. The latter value corresponds to high-strength steel (for example, U10A).

A series of computational calculations consisted of 8 cases. The cases are grouped in such a way that they simultaneously include the calculation of a homogeneous and gradient sample. For example, case 1.1 means that a simulated impact plate on a homogeneous sample with an initial velocity $225 \mathrm{~m} / \mathrm{s}$ and case 1.2 - a shock plate with the same velocity on a FGM sample. The remaining 6 cases are similarly indicated. 


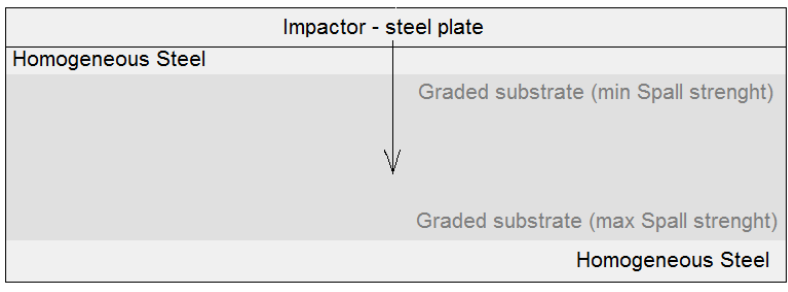

Fig. 4. FGM-sample before impact. Gray shade allocated gradient substrate in the sample. Gray color is allocated homogeneous steel. The velocity vector is directed downward.

Table 1 shows the calculation cases, including the types of samples and the initial velocity of the impactor.

Table 1. Research objects.

\begin{tabular}{|c|l|c|}
\hline Case & \multicolumn{1}{|c|}{ Sample } & $\begin{array}{c}\text { Initial } \\
\text { Velocity of } \\
\text { impactor }\end{array}$ \\
\hline $\begin{array}{l}\text { Case } 1.1 \\
\text { Case } 1.2\end{array}$ & $\begin{array}{l}\text { Steel sample } \\
\text { FGM sample } \\
{\left[\frac{2}{3} \sigma_{k}^{\uparrow}, A_{p}\right]}\end{array}$ & $225 \mathrm{~m} / \mathrm{s}$ \\
\hline $\begin{array}{l}\text { Case } 2.1 \\
\text { Case } 2.2\end{array}$ & $\begin{array}{l}\text { Steel sample } \\
\text { FGM sample }\end{array}$ & $250 \mathrm{~m} / \mathrm{s}$ \\
\hline $\begin{array}{l}\text { Case } 3.1 \\
\text { Case } 3.2\end{array}$ & $\begin{array}{l}\text { Steel sample } \\
\text { FGM sample }\end{array}$ & $275 \mathrm{~m} / \mathrm{s}$ \\
\hline $\begin{array}{l}\text { Case } 4.1 \\
\text { Case } 4.2\end{array}$ & $\begin{array}{l}\text { Steel sample } \\
\text { FGM sample }\end{array}$ & $300 \mathrm{~m} / \mathrm{s}$ \\
\hline
\end{tabular}

The deformation patterns and the areas of destruction of the barriers were analysed and the time formation foci destruction and the further spread of ones in the barriers were monitored. The profiles of the velocity of the free surface, time dependences of the damage and hydrostatic pressure at the control points are computed.

Fig. 5 shows the homogeneous barrier after the impact. The figure illustrates the samples at $20 \mu \mathrm{s}$. In the first two cases, material destruction and plastic deformation of the barriers in the axial and radial directions were took place. In the last two cases, separation of the spall plate from the sample was noted.

The thickness of the spall plate and the initial thickness of the impactor were approximately the same. Spall plate surface was smooth. In all four cases, foci of destruction appear on a $2 \mu$ s process. In the first two cases, they were localized, and in the last two cases they transformed into a macro crack.

Fig. 6 shows the final configuration FGM-samples. Comparing Fig. 5 and 6 can immediately detect differences. In the case 2.1 , the foci of destruction were less than in case 1.1. They were located in a gradient substrate in V-shaped way. The pattern of destruction of a homogeneous and gradient sample in case 1.1 and 2.2 has much in common. In both cases a macro cracks were detected. As seen from Fig.6, presence gradient substrate saved barrier against spalling not eased by the macro crack formation at $275 \mathrm{~m} / \mathrm{s}$ (see case 3.2). In the latter case (case 4.2), the formation of new free surfaces took place in the barrier and led to the separation of the spall plate. However, the fracture patterns in cases 4.1 and 4.2 were somewhat different. In case 4.2, three macro cracks in a gradient substrate were located. It should be noted that the new free surface was less developed. Thus, the impact resistance of a gradient sample is much greater.
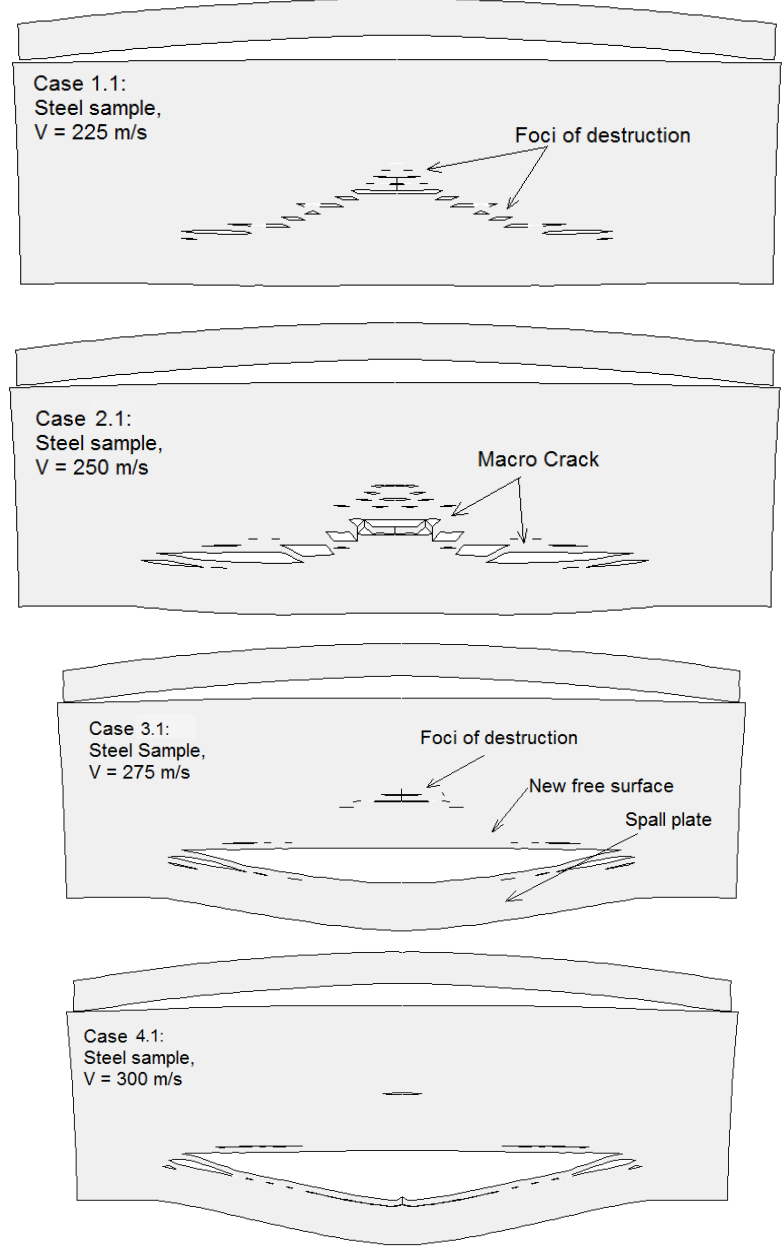

Fig. 5. Steel samples after impact at $t=0.20 \mu \mathrm{s}$. Arrows indicate foci of destruction, new free surfaces, macro cracks and spall plate.
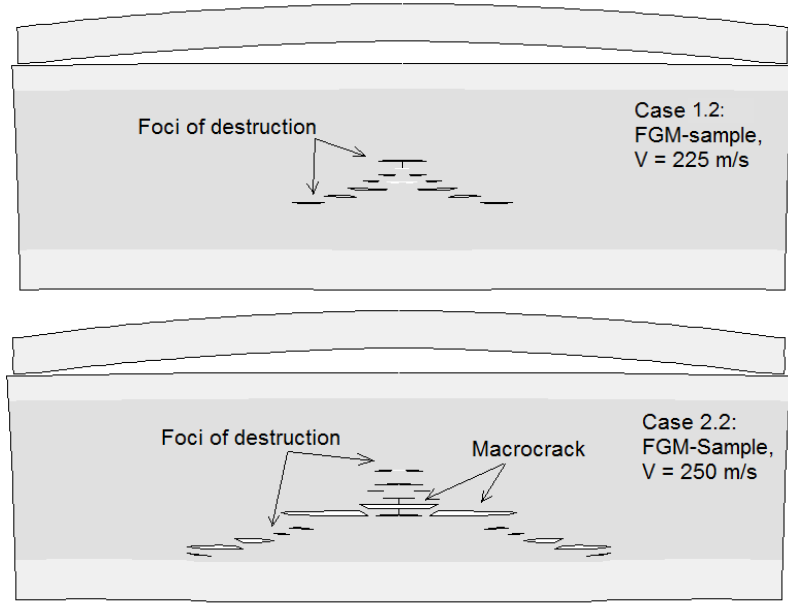

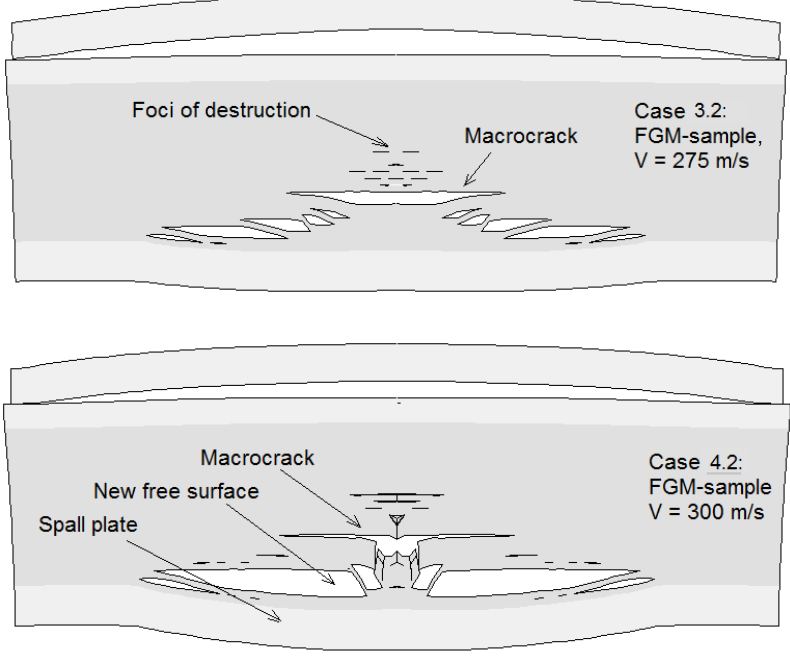

Fig. 6. FGM-samples after impact at $t=0,20 \mu$ s. Gray shade allocated gradient substrate in the sample.

As can be seen from Fig. 7, the free surface profiles for cases 4.1 and 2.2 are different. From the free surface velocity profile, we can get information like HEL (Hugoniot Elastic Limit) velocity, pullback velocity and so on. But this is not discussed in this research.

In those cases where the spall plate was formed, the free velocity profile was as profile in case 4.1. For homogeneous samples this is cases 4.1 and 3.1. For FGM-samples this is only the last case 4.2 (not shown in figure). The output of the shock wave on the free surface is fixed in all cases. This occurred with the $2 \mu \mathrm{s}$ through $4 \mu \mathrm{s}$. After the $20 \mu \mathrm{s}$, the velocity tended to zero. In some cases, the velocity profile repeated the initial compression pulse.

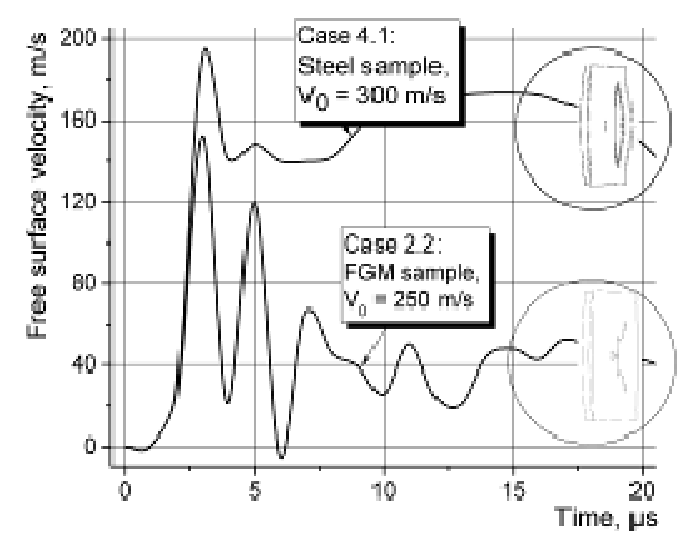

Fig. 7. Free surface profile for Case 4.1 and Case 2.2.

Fig. 8 shows the time dependences versus the hydrostatic pressure at the control points for case 4.1. Of all the cases the pressure profiles were similar. Values are obtained only for control points located in a gradient substrate. The closest point to the contact zone of the "impactor - target" was the control point 5 . The interest is represented by the behaviour of the curves up to $5 \mu \mathrm{s}$, because after the curves faded out. The values of the peak pressures were different for each computational. The maximum value is fixed at the control point 5 in the first microsecond. The minimum pressure is fixed at point 2 between the second and third microseconds.

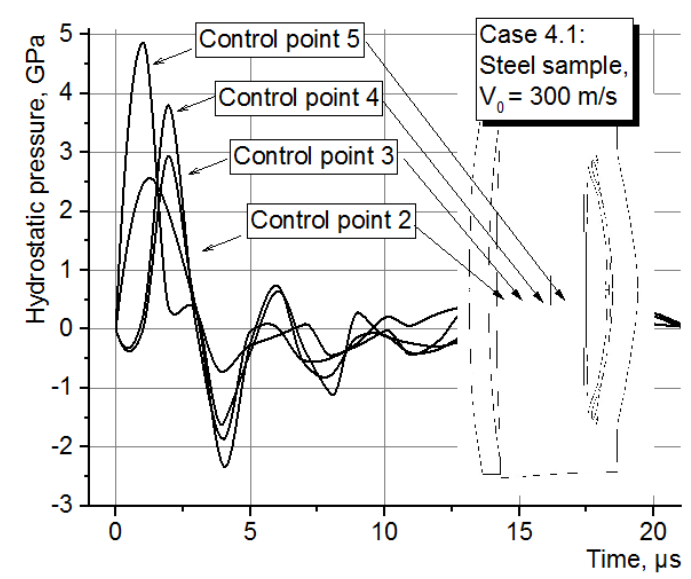

Fig. 8. Damage parameter Vs. Time. The arrows indicate the approximate location of the control points. The exact location is mentioned in section 4 .

In the case 4.1 it is $4.8 \mathrm{GPa}$ and $-2.4 \mathrm{GPa}$, respectively. Of all the cases, after $3 \mu \mathrm{s}$, negative pressures due to tensile stresses are noted. It follows that in the range from 0.5 to $5 \mu \mathrm{s}$, the graded substrate undergoes extreme loads. The values of peak pressures were from $1.39 \mathrm{GPa}$ to $5.03 \mathrm{GPa}$, with the maximum values noted in case 4.1 .

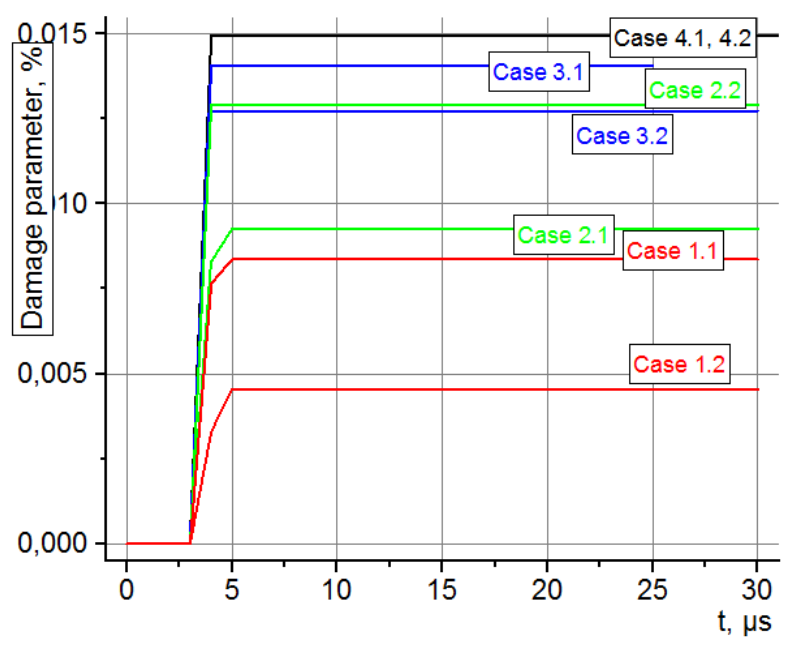

Fig. 9. Hydrostatic pressure vs. Time.

The further interesting and important point to note concerns the destruction of the samples. Fig. 9 illustrates the time dependences of the material on damage parameter. For the first time, the damage parameter was used in [5].

It is seen that the destruction process starts after $2 \mu \mathrm{s}$ and ends at $5 \mu \mathrm{s}$ for all cases. The zone of destruction consists of formed cracks in the middle part of the barriers, foci of destruction along the macro cracks, etc. Minimal material damage was fixed in case 1.2 and the maximum material damage was in cases 4.2 and 4.1. This was 4 and $15 \%$, respectively. The amount of destruction in case 4.2 was greater than in case 4.1 due to foci of destruction in the middle part of the barrier. 
The difference in the material of destruction between cases 1.1 and case 1.2 was almost 2 times. The curves for case 3.1 and case 2.2 are coincided. The increase in the damage parameter after $10 \mu$ s explains the formation of foci of destruction along the spall plate. In five cases, after $5 \mu \mathrm{s}$, the graphics were straight lines. It should be noted that the amount of destruction in FGM samples has always been less than in the homogeneous ones except two last cases.

\section{Conclusion}

Numerical simulation have revealed major features of the process of destruction of homogeneous and graded samples at impact plate. Destruction patterns were formed into barriers from 3 to $12 \mu \mathrm{s}$. Spall plate separation from the sample observed after $10 \mu \mathrm{s}$. Before the failure, the impactors and barriers were deformed and foci of destruction did not appear in impactor. In the range of initial velocities from 250 to $275 \mathrm{~m} / \mathrm{s}$, the presence of a graded substrate prevented from spallation. At velocity of $300 \mathrm{~m} / \mathrm{s}$, the presence of a gradient substrate did not prevent the spall, but only increased the time of destruction of the sample. The surface layer of the gradient substrate was destroyed little. The free surface velocity profiles corresponded to the fracture process and were not discussed in detail in current research.

The hydrostatic pressure in the graded substrate and the homogeneous barrier were quantified. Pressure assumed the maximum and minimum value from 0.5 to 5 $\mu$ s of impact load. After $5 \mu$ s pressure was damped. At the same time, foci of destruction and macro cracks formed in the barriers only. The difference between the peak pressures in the graded substrate and in steel barrier was not significant. The pressure level in the barriers was medium (approximately $5 \mathrm{GPa}$ ). The amount of destruction in the gradient substrate was only less than in the homogeneous barrier, except for the 4.2 case. Most of all, this manifested itself at low velocity. The zones of destruction consisted of foci of destruction in the middle part of the barriers, new free surfaces, etc. and were formed up to $4 \mu$ s of the impact process. It can be argued that the time dependences of damage materials were stepwise form.

Thus, the gradient substrate increased the shock resistance of the barrier to $275 \mathrm{~m} / \mathrm{s}$. Current research allowed to formulate a number of new tasks, which the objects of study are unidirectional FGM. It is reasonable to reduce the thickness of the gradient substrate by a reasonable amount and at the same time to increase the spall strength at the site of the proposed spall. Reducing thickness of the substrate may at $1 / 3$ or $1 / 4$ of the original, but the prescription composition should remain the same. Increasing the value of spall strength can be up to $4 \mathrm{GPa}$. These results will be further developed in another research.
This work was partially funded by the Russian Foundation for Basic Research, the grants No. 16-08-00037a.

\section{References}

1. B. Lyukshin, A. Gerasimov, R. Krektuleva, P. Lyukshin Modeling of physics and mechanical processes in heterogeneous constructions. Novosibirsk. (p.272, Publishing House of the SB $R A S, 2001)$

2. L. Lu, M. Chekroun M., O. Abraham, V. Maupin, G. Villain NDT\&E International, Vol. 44, Issue 2, pp. 169-177 (2011)

3. M. Yu. Orlov, V. Glazyrin, and Yu. Orlov AIP Conference Proceedings 1893, 030133 (2017)

4. Johnson G, Colby D and Vavrick D Int. J. Numer. Methods Engng. 141865 (1979)

5. Gerasimov A et al Theoretical and experimental studies of high-speed interaction of bodies (Tomsk State University Publishing House, 2007)

6. V. Fomin, A. Gulidov, G. Sapozhnikov et al. Highspeed interaction of bodies Novosibirsk (Publishing House of the SB RAS, 1999)

7. Lars Olovsson, Jome Limido, Jean-Luc Lacome, Arve Grornsund Hanssen and Jacques Petit // EPJ Web of Conferences 94, 04050 (2015)

8. K. Stanyukovich et al Physics of Explosion (Moscow: Nauka 1975)

9. Wilkins M Computational Methods in Hydrodynamics (Publishing House Mir, 1967)

10. V. Trushkov, L. Efremova, A. Korneev Combustion, Explosive, and Shock wave, 23(2), pp. 217-222 (1987)

11. G. Kannel, V. Fortov, S. Razorenov, A. Utkin, Shock-wave phenomena in condensed media (1996)

12. S.J. Fensin, E.K. Walker, E.K. Cerreta and G.T. Gray III EPJ Web of Conferences 94, 02010 (2015) 